\title{
A simplified method for the computation of correlation effects on the band structure of semiconductors
}

\author{
U. Birkenheuer ${ }^{1}$, P. Fulde ${ }^{1}$ and H. Stoll ${ }^{2}$ \\ ${ }^{1}$ Max-Planck-Institut für Physik komplexer Systeme, Nöthnitzer \\ Straße 38, 01187 Dresden, Germany \\ ${ }^{2}$ Universität Stuttgart, Pfaffenwaldring 57, 70550 Stuttgart, Germany
}

(May 1, 2019)

\begin{abstract}
We present a simplified computational scheme in order to calculate the effects of electron correlations on the energy bands of diamond and silicon. By adopting a quasiparticle picture we compute first the relaxation and polarization effects around an electron set into a conduction band Wannier orbital. This is done by allowing the valence orbitals to relax within a self-consistent field (SCF) calculation. The diagonal matrix element of the Hamiltonian leads to a shift of the center of gravity of the conduction band while the off-diagonal matrix elements result in a small reduction of the conduction-electron band width. This calculation is supplemented by the computation of the loss of ground state correlations due to the blocked Wannier orbital into which the added electron has been placed. The same procedure applies to the removal of an electron, i.e., to the valence bands. But the latter have been calculated previously in some detail and previous results are used in order to estimate the energy gap in the two materials. The numerical data reported here shows that the methods works, in principle, but that also some extension of the scheme is necessary to obtain fully satisfactory results.
\end{abstract}

Dedicated to J.-P. Malrieu on the occasion of his 60 th birthday.

\section{INTRODUCTION}

Reliable energy-band calculations of solids remain a major topic of modern condensed-matter theory. The problem is a very old one and has been treated on various levels of accuracy and sophistication. Originally the calculations were based on a one-electron theory, i.e., many-body effects were simply neglected or indirectly accounted for ${ }^{1}$. A good choice of model potentials was crucial for the determination of energy bands, e.g., of semiconductors or metals. After the development of density functional theory (DFT) the field obtained an immense impact. Although the theory is designed for ground-state properties it has been also used for the determination of energy bands. Within the local density approximation (LDA) density functional theory could be cast into the form of the Kohn-Sham equation ${ }^{2}$ containing a selfconsistent potential which is free of model-dependent assumptions. The eigenvalues of that equation are simply interpreted as representing the energy bands of the system. The computational efforts were significantly reduced and simplified by the development of linearized methods $^{3}$. The results agreed often surprisingly well with experiments indicating partial error cancellations. In cases where the agreement is poor it is difficult to make improvements which are free of arbitrariness. For example, for strongly correlated electrons the $\mathrm{LDA}+U$ method has been applied ${ }^{4}$ where the intra-atomic correlations are described by a Hubbard interaction term with an on-site Coulomb integral $U$. Thereby the problem of double counting of the interactions must be carefully considered. More recently the LDA $+U$ has been combined with the dynamical mean-field theory $(\mathrm{DMFT})^{5}$, an extension of the coherent potential approximation (CPA) to include the effects of dynamical fluctuations (dynamical $\mathrm{CPA})^{6}$, or the so-called GW method ${ }^{7,8}$.

One of the shortcomings of the LDA when applied to semiconductors or insulators is the underestimation of the size of the gap between the valence and conduction bands. It is of no surprise that the LDA fails here because the correlation cloud around an electron set into the conduction band differs strongly from that of electrons in the charge-neutral ground state. While the former includes a long-ranged polarization cloud the latter consists of van der Waals fluctuations. But those two different physical situations cannot be described by a single self-consistent potential depending on the density only as assumed in the LDA. Therefore one would like to apply quantumchemical methods in order to calculate energy bands of semiconductors. They allow for controlled approximations and can be done at different levels of accuracy.

Calculations based on quantum-chemical methods require a self-consistent field (SCF) or Hartree-Fock (HF) energy-band calculation as a starting point. With the CRYSTAL program package ${ }^{9}$ such calculations can be done. Furthermore, because of the local character of the correlation hole around an electron one also needs to know the Wannier functions for a given semiconductor. Correlation effects arise from exciting electrons out of those localized states. Wannier functions can be ob- 
tained either from the CRYSTAL program package ${ }^{9}$ or from an alternative code called WANNIER ${ }^{10}$. While it is straight forward to determine Wannier orbitals for valence bands it is much more difficult to do the same for conduction bands, due to their weaker localization and the frequently encountered mixing of the low-lying conduction bands with energetically higher-lying bands. But with a proper band disentanglement procedure this can be achieved too ${ }^{11}$. It would be preferable to excite electrons out of local non-orthogonal orbitals ${ }^{12}$ implying the use of a different set of orbitals for a SCF and a correlation calculation. But in that case one cannot use standard quantum chemical programs but rather has to develop new ones.

In order to calculate energy bands for semiconductors with quantum chemical methods one can proceed along two different routes. One starts from the quasiparticle picture ${ }^{13,14}$. The real part of the excitation energy is assumed to be much larger than the imaginary part so that the life time of the excitation may be neglected. In that case one identifies the most important microscopic processes which generate the correlation hole around an electron or hole added to the system and diagonalizes the Hamiltonian in that particular subspace. The latter includes the additional electron or hole (projection method). A related method is based on an effective Hamiltonian and has been exploited by Malrieu and coworkers $^{15,16}$ and others ${ }^{17}$. The second route consists of calculating Green's functions ${ }^{18-22}$, the poles of which determine the excitation spectrum. In both cases an incremental scheme ${ }^{23}$ can be applied when the correlation hole is constructed around the added particle or hole. A number of high-quality calculations have been performed for the valence energy bands, e.g., of diamond and silicon $^{14,24,25}$. However, one is still not able to calculate the conduction bands (and thus the energy gap) of these materials in the same way, even with our new embedding tool $^{26,27}$ Convergence of the approach to an accuracy better than $0.5 \mathrm{eV}$ seems presently hard to achieve.

Here we want to suggest an alternative scheme for energy bands which is based on the quasiparticle approach. It uses a division of the correlation effects caused by the added particle (i.e., electron or hole) into two parts. One concerns polarization and relaxation around the particle and another the loss of ground-state correlations associated with its presence. A splitting of this form was studied previously for a simplified model which allowed for a nearly analytic treatment of those correlation contributions to the energy bands ${ }^{28}$. Here we want to demonstrate that it can be used also for more accurate results based on an ab initio Hamiltonian for a respectable basis set. Calculations of this kind seem to be much easier than previously used computational schemes for dealing with the Hamiltonian.

The numerical results which we shall present here require further work and improvements. That is related to the rather diffuse form of the Wannier functions for conduction electrons in the covalent systems diamond and silicon. But it is also demonstrated that the improvements are straight forward.

\section{DESCRIPTION OF THE METHOD}

We assume that the Hamiltonian is written for a basis set consisting of Gauss-type orbitals (GTO's). Furthermore, we assume that the Hartree-Fock (HF) or selfconsistent field ground-state wave function $\left|\Phi_{\mathrm{SCF}}\right\rangle$ of the $N$-electron system has been determined. For the elemental semiconductors we are interested in, this can be achieved by using the program package $\mathrm{CRYSTAL}^{9}$. When an electron with momentum $\mathbf{k}$ and spin $\sigma$ is added to the energy band $\nu$ the $(N+1)$-particle state is

$$
\left|\Phi_{\nu \sigma}^{N+1}(\mathbf{k})\right\rangle=c_{\mathbf{k} \nu \sigma}^{+}\left|\Phi_{\mathrm{SCF}}\right\rangle
$$

Similarly, when an electron is removed from the system the state is

$$
\left|\Phi_{\nu \sigma}^{N-1}(\mathbf{k})\right\rangle=c_{\mathbf{k} \nu \sigma}\left|\Phi_{\mathrm{SCF}}\right\rangle
$$

Next we want to express the Bloch states as superposition of Wannier states. From the occupied HF orbitals one can construct Wannier orbitals without problems, e.g., by using the Wannier-Boys localization method ${ }^{29}$ implemented in CRYSTAL $^{9}$. For the virtual space this is more difficult. The required procedure is described in Ref. ${ }^{11}$ to which we refer. In the following we assume that those orbitals have been determined and that the corresponding creation (destruction) operator are $c_{\mathbf{R} n \sigma}^{+}$ $\left(c_{\mathbf{R} n \sigma}\right)$. Here $\mathbf{R}$ denotes the lattice vector of the unit cell at which the Wannier orbital is centered while $n$ is an intracell index. One can imagine the Wannier orbitals as representing the bonding and antibonding orbitals in the diamond structure of the elemental semiconductors. We write

$$
\left|\Phi_{\nu \sigma}^{N+1}(\mathbf{k})\right\rangle=\frac{1}{\sqrt{N_{c}}} \sum_{\mathbf{R} n} \alpha_{\nu n}(\mathbf{k}) e^{i \mathbf{k} \mathbf{R}} c_{\mathbf{R} n \sigma}^{+}\left|\Phi_{\mathrm{SCF}}\right\rangle
$$

and similarly for $\left|\Phi_{\nu \sigma}^{N-1}(\mathbf{k})\right\rangle$. Here $N_{c}$ is the number of unit cells. The conduction-band energies in Hartree-Fock approximation can be expressed in terms of local matrix elements as

$$
\epsilon_{\mathbf{k} \nu}^{\mathrm{SCF}}=\sum_{\mathbf{R}} \sum_{n n^{\prime}} \alpha_{\nu n}(\mathbf{k}) \alpha_{\nu n^{\prime}}^{*}(\mathbf{k}) e^{i \mathbf{k} \mathbf{R}} H_{\mathbf{R} n n^{\prime}}^{\mathrm{SCF}}
$$

with matrix elements

$$
\begin{aligned}
H_{\mathbf{R}-\mathbf{R}^{\prime}, n n^{\prime}}^{\mathrm{SCF}}= & \left\langle\Phi_{\mathrm{SCF}}\left|c_{\mathbf{R}^{\prime} n^{\prime} \sigma} H c_{\mathbf{R} n \sigma}^{+}\right| \Phi_{\mathrm{SCF}}\right\rangle \\
& -\delta_{\mathbf{R} \mathbf{R}^{\prime}} \delta_{n n^{\prime}} E_{0}^{\mathrm{SCF}} .
\end{aligned}
$$

Here $E_{0}^{\mathrm{SCF}}$ is the Hartree-Fock ground-state energy. When the electronic correlations are included, the Hartree-Fock excitations become quasiparticle excitations. For relatively weakly correlated electron systems 
one may assume that there is still a one-to-one correspondence of the Hartree-Fock and quasiparticle excitations. In this case the energy bands describe the dispersion of the quasiparticles. Life-time effects are neglected. Thus we write for the correlated $(N+1)$-electron state

$$
\begin{aligned}
\left|\Psi_{\nu \sigma}^{N+1}(\mathbf{k})\right\rangle & =\tilde{\Omega}\left|\Phi_{\nu \sigma}^{N+1}(\mathbf{k})\right\rangle \\
& =\frac{1}{\sqrt{N_{c}}} \sum_{\mathbf{R} n} \alpha_{\nu n}(\mathbf{k}) e^{i \mathbf{k R}} \tilde{\Omega} c_{\mathbf{R} n \sigma}^{+}\left|\Phi_{\mathrm{SCF}}\right\rangle
\end{aligned}
$$

where the wave- or Möller operator $\tilde{\Omega}$ transforms $\left|\Phi_{\nu \sigma}^{N+1}(\mathbf{k})\right\rangle$ into $\left|\Psi_{\nu \sigma}^{N+1}(\mathbf{k})\right\rangle$. The correlated conduction bands are given by

$$
\epsilon_{\nu \sigma}(\mathbf{k})=\frac{\left\langle\Phi_{\nu \sigma}^{N+1}(\mathbf{k})\left|\tilde{\Omega}^{+} H \tilde{\Omega}\right| \Phi_{\nu \sigma}^{N+1}(\mathbf{k})\right\rangle}{\left\langle\Phi_{\nu \sigma}^{N+1}(\mathbf{k})\left|\tilde{\Omega}^{+} \tilde{\Omega}\right| \Phi_{\nu \sigma}^{N+1}(\mathbf{k})\right\rangle}-E_{0}
$$

where $E_{0}$ is the exact, i.e., correlated ground-state energy. In terms of cumulants this can be rewritten as

$$
\epsilon_{\nu \sigma}(\mathbf{k})=(H \mid \Omega)_{\mathbf{k} \nu \sigma}-E_{0}
$$

where the notation

$$
(A \mid B)_{\mathbf{k} \nu \sigma}=\left\langle\Phi_{\nu \sigma}^{N+1}(\mathbf{k})\left|A^{+} B\right| \Phi_{\nu \sigma}^{N+1}(\mathbf{k})\right\rangle^{c}
$$

has been used with the index $c$ indicating that the cumulant has to be taken. For cumulants and their properties we refer to Ref. 30 where also the original literature is cited. The operator $\Omega$ is the cumulant wave operator, i.e., it is defined only in connection with the form (9). It is customary to write $\Omega=1+S$ where $S$ is a scattering matrix. By decomposing the Hamiltonian $H=H_{\mathrm{SCF}}+H_{\text {res }}$ we obtain for the correlation correction of the conduction bands

$$
\begin{aligned}
\epsilon_{\nu \sigma}(\mathbf{k})= & \epsilon_{\nu \sigma}^{\mathrm{SCF}}(\mathbf{k})+\Delta \epsilon_{\nu \sigma}(\mathbf{k}) \\
\Delta \epsilon_{\nu \sigma}(\mathbf{k})= & \sum_{\mathbf{R}} \sum_{n n^{\prime}} \alpha_{\nu n}(\mathbf{k}) \alpha_{\nu n^{\prime}}^{*}(\mathbf{k}) e^{i \mathbf{k R}} \\
& \left(c_{o n^{\prime} \sigma}^{+} \mid H_{\mathrm{res}} S c_{\mathbf{R} n \sigma}\right) .
\end{aligned}
$$

The round brackets $(A \mid B)$ refer to the cumulants

$$
(A \mid B)=\left\langle\Phi_{\mathrm{SCF}}\left|A^{+} B\right| \Phi_{\mathrm{SCF}}\right\rangle^{c} .
$$

Note that the cumulant $\left(c_{\mathbf{0} n^{\prime} \sigma}^{+} \mid H_{\text {res }} S c_{\mathbf{R} n \sigma}^{+}\right)$contains a term $E_{\text {corr }}=E_{0}-E_{0}^{\mathrm{SCF}}$ in form of a subtraction which describes the correlation energy of the ground state of the $N$-electron system. It changes when differently localized Wannier functions are used. Yet, since Wannier functions provide a complete basis, the shifts $\Delta \epsilon_{\nu \sigma}$ remain the same after summation of all relevant contributions according to (10).

For systems like the elemental semiconductors it suffices to include in $S$ one- and two-particle excitations. Thereby, the annihilation operators $c_{\mathbf{R} n \sigma}$ refer to the occupied Wannier orbitals of the $(N+1)$-electron system while the number of the local creation operators $a_{\mathbf{R} \tau \sigma}^{+}$ depends on the size of the basis set. The latter are, e.g., the ones for GTO's with the GTO being centered at site (or bond) $\tau$ of the unit cell R. Note that due to the use of cumulants only connected operator products contribute. Thus the following general ansatz for the scattering matrix $S$ holds

$$
\begin{aligned}
S & =\sum_{\mathbf{R}_{1} \mathbf{R}_{2}} \sum_{\tau n} \sum_{\sigma \sigma^{\prime}} \eta_{\mathbf{R}_{1} \mathbf{R}_{2}}^{\tau n} a_{\mathbf{R}_{1} \tau \sigma}^{+} c_{\mathbf{R}_{2} n \sigma} \\
& +\sum_{\mathbf{R}_{1} \mathbf{R}_{2} \mathbf{R}_{3} \mathbf{R}_{4}} \sum_{\tau \tau^{\prime} n n^{\prime}} \sum_{\sigma \sigma^{\prime}} \eta_{\mathbf{R}_{1} \mathbf{R}_{2} \mathbf{R}_{3} \mathbf{R}_{4}}^{\tau \tau^{\prime} n n^{\prime}} \\
& \cdot a_{\mathbf{R}_{1} \tau \sigma}^{+} a_{\mathbf{R}_{2} \tau^{\prime} \sigma^{\prime}}^{+} c_{\mathbf{R}_{3} n \sigma^{\prime}} c_{\mathbf{R}_{4} n^{\prime} \sigma}
\end{aligned}
$$

It is advantageous to decompose $S$ into a part which commutes with the added electron $c_{\mathbf{R} n \sigma}^{+}$and a part which does not, i.e.,

$$
S=S_{\pi}+S_{\eta}
$$

with

$$
\left[S_{\pi}, c_{\mathbf{R} n \sigma}^{+}\right]_{-} \neq 0 \text { and }\left[S_{\eta}, c_{\mathbf{R} n \sigma}^{+}\right]_{-}=0 .
$$

The operator $S_{\pi}$ describes the relaxation and polarization around the electron generated by $c_{\mathbf{R} n \sigma}^{+}$while $S_{\eta}$ describes the loss of ground-state correlations due to presence of the extra electron. As pointed out before, the latter results from blocking the orbital occupied by the added electron for excitations out of the ground state. Thus

$$
\begin{aligned}
\left(c_{\mathbf{0} n^{\prime} \sigma}^{+} \mid H_{\mathrm{res}} S c_{\mathbf{R} n \sigma}^{+}\right) & =\left\langle\Phi_{\mathrm{SCF}}\left|c_{\mathbf{0} n^{\prime} \sigma} H_{\mathrm{res}} S_{\pi} c_{\mathbf{R} n \sigma}^{+}\right| \Phi_{\mathrm{SCF}}\right\rangle^{c} \\
& +\left\langle\Phi_{\mathrm{SCF}}\left|c_{\mathbf{0} n^{\prime} \sigma} H_{\mathrm{res}} c_{\mathbf{R} n \sigma}^{+} S_{\eta}\right| \Phi_{\mathrm{SCF}}\right\rangle^{c}
\end{aligned}
$$

The first term, i.e., the polarization and relaxation energy is approximated by a SCF calculation for the $(N+1)$ electron system with an electron frozen in Wannier orbital $(\mathbf{R} n \sigma)$. This can be done by making use of the program package MOLPRO ${ }^{31}$. The energy contribution of the second term is computed by determining those excitations in a calculation of the ground-state correlation energy which are blocked by the creation $c_{\mathbf{R} n \sigma}^{+}$of an electron. Their contribution to the energy can be extracted from a ground-state calculation. The details of this procedure are described in the following subsection. Needless to say that the same prescription applies for calculating the valence band, i.e., when an electron is removed from the $N$-particle ground state.

Although the scheme proposed here consists of several individual steps and may seem cumbersome from a technical point of view, the present approach is conceptionally much simpler than other $a b$ initio methods for band structures ${ }^{14,21,22,24,25,32}$. It is designed such that it only requires Hartree-Fock type calculations and some small configuration interaction (CI) correlation calculations to estimate the correlation effects on the energy bands. Both type of calculations can be performed routinely and without much computational effort by standard quantum chemical program packages. 


\section{COMPUTATIONS AND RESULTS}

In a series of papers ${ }^{14,24,25}$, we presented results for the influence of correlation effects on the valence band structure of diamond and silicon. These results were obtained using a local Hamiltonian formalism in finite cluster calculations for the neutral $N$-particle state and cationic $(N-1)$-particle hole states. Unfortunately, the structure of the conduction band is not accessible this way, since an additional electron in negatively charged $(N+1)$-particle states of finite $\mathrm{C}$ and $\mathrm{Si}$ clusters is not bound. Therefore, only rough estimates of the band gaps in diamond and silicon could be given in Ref. 25, assuming that the energetic effect of the polarization cloud around a conduction band electron would be approximately the same as that around a valence-band hole.

In the meantime, we adapted our local Hamiltonian formalism to the treatment of conduction bands, by extracting the localized conduction band Wannier functions (WF) from periodic Hartree-Fock (HF) calculations ${ }^{29,33}$. The same calculations are used to generate embedding potentials which describe the influence of the infinite bulk surroundings on finite cluster models ${ }^{26}$. Results for the band structure of diamond ${ }^{34}$ and hydrogen fluoride chains $^{35}$ with this approach are under preparation in our laboratory.

As an alternative, we present here results for the diamond and silicon conduction bands using the simplified method described in the previous section. Thereby, instead of generating local matrix elements of the system's Hamiltonian between localized multi-determinant conduction band states, we start from the simple physical picture of a polarization cloud forming around an added conduction-band electron and calculate the (static) polarization and relaxation effects around this electron when it is placed in a frozen conduction band WF. This yields localized quasi-particle states which we can use as a basis for the final diagonalization of the Hamiltonian. This in turn should lead to a realistic quantitative description of correlation effects on the conduction bands. The advantage over the local Hamiltonian approach lies in the facts that by freezing the conduction band WF, we do not encounter stability problems, and that for the determination of the static (rather than dynamic) polarization cloud self-consistent field (SCF) calculations (rather than correlated calculations) are sufficient. It is clear, on the other hand, that the computational simplification cannot be achieved without introducing additional approximations: the difference between static and dynamic relaxation/polarization effects is neglected and, more importantly, correlation effects beyond relaxation/polarization are also neglected at this stage. The most important of the latter effects is the loss of groundstate correlation when introducing an electron in the conduction band, i.e., the exclusion effect due to the nonavailability of excitations from occupied orbitals of the neutral ( $N$-electron) system into the conduction band
Wannier spin orbital occupied in the $(N+1)$-electron system. However, the leading term of this exclusion effect on the diagonal matrix element can easily be recovered in a small configuration-interaction (CI) calculation, restricted to excitations from the occupied SCF orbitals of the closed-shell $N$-electron system into a single localized conduction band WF, without abandoning the advantages of computational simplicity.

Following the above strategy, we performed the following detailed computational steps:

1) We started from periodic $\mathrm{HF}$ calculations for bulk diamond and silicon. The CRYSTAL200x program $^{33}$ was used for that purpose, a pre-cursor of the most recent public version of the CRYSTAL program package ${ }^{9}$ which already allowed for localization. The basis sets used were of valence-triplezeta quality, with a single $d$ polarization function, optimized for diamond ${ }^{36,37}$ and silicon ${ }^{38}$. In the case of $\mathrm{Si}$, the $[1 s 2 s 2 p]$ core was simulated by an effective core potential ${ }^{38}$. These calculations provide the $\mathrm{HF}$ band structure of the conduction (and valence) bands.

2) The Wannier-Boys algorithm ${ }^{29,39}$ is used to optimize the unitary band mixing matrix of the multiband Wannier transformation. It is applied separately to the valence band and conduction band Bloch functions to generate localized occupied and virtual Wannier functions. In the case of $\mathrm{Si}$, the conduction band states had to be disentangled from those of the higher-lying unoccupied bands before they could be localized ${ }^{11}$. The mean width $\sqrt{\left\langle(\mathbf{r}-\langle\mathbf{r}\rangle)^{2}\right\rangle}$ of the resulting virtual Wannier functions is found to be in the order of 1.5-2 times the bond length in the elemental semiconductor.

3) The data of steps 1 and 2 were transferred via our CRYSTAL-MOLPRO interface ${ }^{27}$ to the molecular program MOLPRO ${ }^{31,40}$ and are used as input for generating embedded $\mathrm{C}_{8(18)}$ and $\mathrm{Si}_{8(18)}$ clusters ${ }^{34}$. Here, the central $\mathrm{C}-\mathrm{C}$ or $\mathrm{Si}-\mathrm{Si}$ bond and its six nearest-neighbor bonds inside the $\mathrm{C}_{8}\left(\mathrm{Si}_{8}\right)$ kernel are treated as active, while the basis functions from the surrounding shell of $18 \mathrm{C}(\mathrm{Si})$ atoms are only added to assist in the representation of the localized occupied and virtual orbitals of the kernel. These $\mathrm{C}_{26}$ and $\mathrm{Si}_{26}$ support clusters are sufficient to provide good representations of the localized Wannier functions inside the kernel. Upon projection onto the support clusters the norms of the virtual Wannier functions reduce by $1-2 \%$ only. For the generation of the local virtual basis functions a compact variant ${ }^{26}$ of the projected atomic orbital construction $^{41}$ is used. The Hartree-Fock potential of all non-active occupied C-C/Si-Si orbitals on the cluster and the surrounding solid (together with the nuclei there) is incorporated in the embedding potential. 
4) The HF reference data for matrix elements between localized $(N+1)$-particle states can now be generated according to

$$
\left\langle\Phi_{i}^{N+1}|H| \Phi_{j}^{N+1}\right\rangle-\left\langle\Phi_{\mathrm{SCF}}|H| \Phi_{\mathrm{SCF}}\right\rangle \delta_{i j}
$$

where $\Phi_{\mathrm{SCF}}$ is the HF wave function of the neutral $\mathrm{C}_{8(18)}$ or $\mathrm{Si}_{8(18)}$ cluster, and $\Phi_{i}^{N+1}$ is the same wave function with a localized (anti-bonding) conduction band WF (from step 2) added at bond $i$. The latter describes the local HF configurations $c_{\mathbf{R} n \sigma}^{+} \Phi_{\mathrm{SCF}}$ introduced in Eq. (3).

5) To estimate the correlation effects for the diagonal matrix element $(i=j)$, we first replace $\Phi_{i}^{N+1}$ in Eq. (16) by $\tilde{\Phi}_{i}^{(N+1)}$ where the valence orbitals are allowed to relax and polarize due to the presence of the (fixed) conduction band WF of the $(N+1)$-particle system. This single-determinant wave function serves as a first approximation to the correlated counter parts $\tilde{\Omega} c_{\mathbf{R} n \sigma}^{+} \Phi_{\mathrm{SCF}}$ from Eq. (6).

6) To include the leading term to the loss of ground state correlation for the diagonal matrix element, we further replace $\tilde{\Phi}_{i}^{N+1}$ by $\tilde{\Psi}_{i}^{N+1}$, and $\Phi_{\mathrm{SCF}}$ by $\tilde{\Psi}^{N}$ in Eq. (16), where $\tilde{\Psi}_{i}^{N+1}$ and $\tilde{\Psi}^{N}$ are full CI wave functions in the space of the active valence band orbitals (from step 4 for the N-particle system, and step 5 for the $(\mathrm{N}+1)$-particle system) plus the localized conduction band WF at site $i$. (We also tried including additional excitation channels with one electron in one of the conduction-band WF $j$ adjacent to $i$, but the effect on the numerical results to be described below was negligible $[<0.05$ $\mathrm{eV}]$.

7) In order to get the leading term for the correlation effects on the off-diagonal matrix element between nearest-neighbor bond sites $i \neq j$, we focus on the relaxation and polarization effect and approximately evaluate

$$
\begin{aligned}
\left\langle\tilde{\Phi}_{i}^{N+1}|H| \tilde{\Phi}_{j}^{N+1}\right\rangle \approx & \left\langle\Phi_{i}^{N+1}|H| \Phi_{j}^{N+1}\right\rangle \\
& +2\left\langle\Phi_{i}^{N+1}|H| \tilde{\Phi}_{j}^{N+1}-\Phi_{j}^{N+1}\right\rangle
\end{aligned}
$$

exploiting the fact that $\tilde{\Phi}_{i}^{N+1}$ and $\tilde{\Phi}_{j}^{N+1}$ are equivalent in diamond lattices. To this end the polarized active valence orbitals $\tilde{\varphi}_{n}$ entering the determinant $\tilde{\Phi}_{j}^{N+1}$ are expanded into the unpolarized ones $\left(\varphi_{n}\right)$ defining $\Phi_{j}^{N+1}$ plus orthogonal relaxation contributions $\omega_{k}$ in the virtual space

$$
\tilde{\varphi}_{n}=\sqrt{1-c_{n}^{2}} \varphi_{n}+c_{n} \omega_{n}
$$

and $\tilde{\Phi}_{j}^{N+1}$ is replaced by a CI wave function with single excitations from the $\varphi_{n}$ into the $\omega_{n}$.
The resulting SCF and correlation contributions are listed in Table 1. The employed Wannier functions are rather compact, and the matrix elements of the local Hamiltonian should decay rapidly with increasing distance of the bond pairs, such that the diagonal and nearest-neighbor off-diagonal terms should suffice to recover most of the correlation effects. Switching to different localized Wannier functions would alter these contributions slightly, but after diagonalization of the resulting Hamiltonian matrix these modifications cancel to a large extent. At present, we do not have any alternative localization scheme for Wannier functions at hand to corroborate this numerically.

The above diagonal correlation contributions lead to a shift of the center of gravity of the band and sum up to $-1.08 \mathrm{eV}$ for diamond and $-0.85 \mathrm{eV}$ for silicon. These energies are significantly smaller in magnitude than the corresponding values found in Ref. 25 for the valence band (relaxation/polarization: $4.6 \mathrm{eV}$ for $\mathrm{C}, 3.4 \mathrm{eV}$ for $\mathrm{Si}$; loss of ground-state correlation: $-0.7 \mathrm{eV}$ for $\mathrm{C},-0.6 \mathrm{eV}$ for $\mathrm{Si}$ ). This finding is in strong contrast to an assumption put forward in Ref. 25, namely that the correlation effects caused by conduction electrons and valence holes are symmetric and presumably has to be attributed to the fact, that the conduction band WFs are much less localized than the valence band WFs. Still, the assumption is bound to hold for long-range polarization effects, since the polarizing charges are of the same magnitude $( \pm e)$, and if we extract the polarization contributions beyond nearest-neighbor bonds (not calculated in the present paper) from the valence-band calculations of Ref. $25(3.5 \mathrm{eV}$ for $\mathrm{C}$, and $2.4 \mathrm{eV}$ for Si, without two- and three-body contributions), we can estimate the total correlation-induced shift of the conduction band center of gravity to $-4.6 \mathrm{eV}$ for $\mathrm{C}$, and $-3.3 \mathrm{eV}$ for $\mathrm{Si}$. A much simpler estimate of the long-range polarization contribution to the shift of the energy bands can be obtained from the classical polarization energy of a dielectric medium outside a sphere of radius $R_{\text {cut }}$ (see Ref. 30)

$$
\Delta \epsilon=-\frac{\epsilon_{0}-1}{2 \epsilon_{0}} \frac{e^{2}}{R_{\mathrm{cut}}} .
$$

with $\epsilon_{0}$ being the dielectric constant of the medium. Using the experimental values for the dielectric constant

TABLE I. Contributions to the diagonal and off-diagonal Hamiltonian matrix elements (in eV) between localized conduction band states in bulk diamond and silicon at various levels of theory (cf. text).

\begin{tabular}{lrrrr}
\hline \hline & \multicolumn{2}{c}{ diagonal } & \multicolumn{2}{c}{ off-diagonal } \\
& diamond & silicon & diamond & silicon \\
\hline Hartree-Fock & 14.77 & 7.07 & 0.23 & 0.20 \\
relaxation/polarization & -1.28 & -0.98 & -0.01 & -0.02 \\
loss of ground-state corr. & 0.20 & 0.13 & & \\
long-range rel./pol. ${ }^{a}$ & -3.5 & -2.4 & & \\
\hline${ }^{a}$ estimate based on the date in Ref. 25 & \\
\hline \hline
\end{tabular}


(5.7 for diamond and 12.0 for silicon $)^{42}$ the above estimates of the long-range polarization contributions correspond to cutoff radii of $1.72 \AA$ for diamond and $2.75 \AA$ for silicon. To gain some insight into the accuracy of the long-range estimates these cutoff radii can be compared to the radii of the 8 atomic cluster kernels (defined here as the distance of the outermost kernel atoms from the center of the kernel). They amount to 1.89 and $2.88 \AA$ for $\mathrm{C}$ and $\mathrm{Si}$, respectively, which is fairly close.

Turning now to the off-diagonal matrix elements between nearest-neighbor bonds we find again here that the correlation-induced changes are much smaller than for the corresponding valence band quantities evaluated in Refs. [ 14, 24] (changes in the matrix elements of 0.7 $\mathrm{eV}$ for $\mathrm{C}$ and $0.8 \mathrm{eV}$ for $\mathrm{Si}$ ). In diamond lattices the nearest-neighbor off-diagonal elements enter with a factor of 8 into the bandwidth at the $\Gamma$-point of the uppermost (lowermost) four valence (conduction) bands and with a factor of 2 into the energy at the top (bottom) of the respective band complex. It is essentially these contribution from the nearest-neighbor matrix elements which is responsible for the total correlation-induced change of the valence-band width (of $\sim 7 \mathrm{eV}$ for both, $\mathrm{C}$ and $\mathrm{Si}^{14,24}$ ). Assuming a similar finding for the conduction band, i.e., that only matrix elements between nearest neighbors are important we would estimate the correlation-induced narrowing of its width to be in the order of $0.1-0.2 \mathrm{eV}$. The rising of its bottom would remain well below 0.05 $\mathrm{eV}$, an almost negligible shift. Yet, this assumption is somewhat unrealistic, because the larger extent of the conduction band WFs will lead to a much longer range of the correlation-induced changes of the local matrix elements than in the valence band case, i.e., to substantial changes far beyond nearest neighbours. This is corroborated by our ongoing study of the conduction bands of diamond by means of the local Hamiltonian approach ${ }^{34}$. Unfortunately, at present we do not have estimated data for these changes available.

Indeed, our estimates for the center-of-gravity shifts (cf. above), when taken together with the top-of-valenceband shifts of Ref. 25 (i.e., $3.90 \mathrm{eV}$ for $\mathrm{C}$ and $2.63 \mathrm{eV}$ for $\mathrm{Si}$ ) would lead to significantly too small band gaps (5.3 $\mathrm{eV}$ vs. $7.3 \mathrm{eV}$ from experiment ${ }^{43}$ for $\mathrm{C}$, and $2.5 \mathrm{eV}$ vs. 3.4 $\mathrm{eV}$ from experiment (direct gap) ${ }^{43}$ for $\mathrm{Si}$ ). They are certainly more accurate than the (too large) HF band gaps of $13.8 \mathrm{eV}$ for $\mathrm{C}$, and $8.4 \mathrm{eV}$ for $\mathrm{Si}^{44}$, but are still not acceptable. More accurate data for the correlation-induced narrowing of the conduction band width are needed, and also the determination of the polarization contributions beyond nearest-neighbor bonds must be improved. Work in these directions is underway in our laboratory.

\section{ACKNOWLEDGEMENT}

We thank Dr. C. Willnauer for a number of discussions concerning numerical results.
${ }^{1}$ see, e.g., W. A. Harrison: Electronic Structure and the Properties of Solids (W. H. Freeman and Company, San Francisco 1980; Dover ed., General Publ. Company, Toronto 1989).

${ }^{2}$ W. Kohn and L. Sham, Phys. Rev. A 140, 1133 (1965).

${ }^{3}$ O. K. Andersen, Phys. Rev. B 12, 3060 (1975).

${ }^{4}$ V. I. Anisimov, J. Zaanen and O. K. Andersen, Phys. Rev. B 44, 943 (1991).

${ }^{5}$ V. I. Anisimov, A. I. Poteryaev, M. A. Korotin, A. O. Anokhin and G. Kotliar, J. Phys. Cond. Matter 9, 7359 (1997); A. I. Lichtenstein and M. I. Katsnelson, Phys. Rev. B 57, 6884 (1998).

${ }^{6}$ Y. Kakehashi, Adv. Phys. 53, 497 (2004).

${ }^{7}$ L. Hedin, Phys. Rev. 139, 3A 796 (1965).

${ }^{8}$ G. Strinati, H. J. Mattausch and W. Hanke, Phys. Rev. B 25, 2867 (1982).

${ }^{9}$ V. R. Saunders, R. Dovesi, C. Roetti, R. Orlando, C. M. Zicovich-Wilson, N. M. Harrison, K. Doll, B. Civalleri, I. J. Bush, Ph. D'Arco, M. Llunell, CRYSTAL2003 User's Manual, Theoretical Chemistry Group, University of Torino (2003).

10 A. Shukla, M. Dolg, H. Stoll and P. Fulde, Chem. Phys. Lett. 262, 213 (1996) and Phys. Rev. B 57, 1471 (1998).

${ }^{11}$ U. Birkenheuer and D. Izotov, Phys. Rev. B 71 (2005), in press, arXiv: cond-mat/0409337.

12 G. Stollhoff and P. Fulde, J. Chem. Phys. 73, 4548 (1980).

${ }^{13}$ S. Horsch, P. Horsch and P. Fulde, Phys. Rev. B 29, 1870 (1984).

14 J. Gräfenstein, H. Stoll and P. Fulde, Phys. Rev. B 55, 13588 (1997).

${ }^{15}$ M. Albrecht, P. Reinhardt and J.-P. Malrieu, Theor. Chim. Ac. 100, 241 (1998).

16 J. Rubio, A. Povill, J.-P. Malrieu and P. Reinhardt, J. Chem. Phys. 107, 10044 (1997), see also P. Reinhardt and J.-P. Malrieu, J. Chem. Phys. 109, 7632 (1998).

17 J. Q. Sun and R. J. Bartlett, J. Chem. Phys. 104, 8553 (1996).

18 J. Linderberg and Y. Öhrn: Propagators in Quantum Chemistry (Academic, London 1973).

19 J. J. Ladik, Phys. Rev. 313, 171 (1999).

${ }^{20}$ E. K. U. Gross, E. Runge and O. Heinonen, Many-Particle Theory (Adam Hilger, Bristol 1991).

${ }^{21}$ M. Albrecht and P. Fulde, phys. stat. sol. (b) 234, 313 (2002).

${ }^{22}$ C. Buth, U. Birkenheuer, M. Albrecht, P. Fulde, Phys. Rev. B , submitted - arXiv: cond-mat/0409078.

${ }^{23}$ H. Stoll, Chem. Phys. Lett. 191, 548 (1992), Phys. Rev. B 46, 6700 (1992), and J. Chem. Phys. 97, 8449 (1992).

${ }^{24}$ J. Gräfenstein, H. Stoll and P. Fulde, Chem. Phys. Lett. 215, 610 (1993).

${ }^{25}$ M. Albrecht, P. Fulde and H. Stoll, Chem. Phys. Lett. 319, 355 (2000).

${ }^{26} \mathrm{U}$. Birkenheuer, in preparation.

${ }^{27}$ C. Roetti, R. Dovesi, M. von Arnim, W. Alsheimer, and U. Birkenheuer, The CRYSTAL-MOLPRO interface, MPIPKS Dresden, 2002. 
${ }^{28}$ W. Borrmann and P. Fulde, Phys. Rev. B 35, 9569 (1987).

${ }^{29}$ C. M. Zicovich-Wilson, R. Dovesi and V. R. Saunders, J. Chem. Phys. 115, 9708 (2001).

${ }^{30}$ P. Fulde: Electron Correlations in Molecules and Solids, Springer Series in Solid-State Sciences, Vol. 100, 3rd ed. (Springer, Berlin 1995).

${ }^{31}$ MOLPRO (Version 2002.6) is a package of ab initio programs designed by H.-J. Werner and P. J. Knowles. The authors are R. D. Amos, A. Bernhardsson, A. Berning, P. Celani, D. L. Cooper, M. J. O. Deegan, A. J. Dobbyn, F. Eckert, C. Hampel, G. Hetzer, P. J. Knowles, T. Korona, R. Lindh, A. W. Lloyd, S. J. McNicholas, F. R. Manby, W. Meyer, M. E. Mura, A. Nicklaß, P. Palmieri, R. Pitzer, G. Rauhut, M. Schütz, U. Schumann, H. Stoll, A. J. Stone, R. Tarroni, T. Thorsteinsson, and H.-J. Werner.

${ }^{32}$ V. Bezugly and U. Birkenheuer, Chem. Phys. Lett. 399, 57 (2004).

${ }^{33}$ V. R. Saunders, R. Dovesi, C. Roetti, M. Causá, N. M. Harrison, R. Orlando, C. M. Zicovich-Wilson, K. Doll, and B. Civalleri, CRYSTAL200x User's Manual, Theoretical Chemistry Group, University of Torino (2001).

${ }^{34} \mathrm{C}$. Willnauer and U. Birkenheuer, in preparation.

${ }^{35}$ V. Bezugly, C. Buth, M. Albrecht, and U. Birkenheuer, in preparation.

36 T. H. Dunning, Jr., J. Chem. Phys. 90, 1007 (1989).

37 The most diffuse $s$ exponent was replaced by 0.201142 , the two outermost $p$ exponents by 0.625572 and 0.324363 ; the $d$ exponent of 0.55 was taken from the corresponding double zeta basis.

38 A. Bergner, M. Dolg, W. Kuechle, H. Stoll, and H. Preuss, Mol. Phys. 80, 1431 (1993).

${ }^{39}$ P. Baranek, C. M. Zicovich-Wilson, C. Roetti, R. Orlando, and R. Dovesi, Phys. Rev. B 64, 125102 (2001).

${ }^{40}$ H.-J. Werner and P. J. Knowles, J. Chem. Phys. 82, 5053 (1985); P. J. Knowles and H.-J. Werner, Chem. Phys. Lett. 115, 259 (1985); H.-J. Werner and P. J. Knowles, J. Chem. Phys. 89, 5803 (1988); P. J. Knowles and H.-J. Werner, Chem. Phys. Lett. 145, 514 (1988); H.-J. Werner and P. J. Knowles, Theor. Chim. Acta 78, 175 (1990).

${ }^{41}$ P. Pulay, Chem. Phys. Lett. 100, 151 (1983).

${ }^{42}$ N. W. Ashcroft, N. D. Mermin, Solid State Physics (Saunders College, Philadelphia, 1976), Tab. 27.3.

${ }^{43}$ R. W. Godby, M. Schlüter, and L. J. Sham, Phys. Rev. B 36, 6497 (1987).

${ }^{44}$ R. Orlando, R. Dovesi, C. Roetti, and V. R. Saunders, J. Phys.: Condens. Matter 2, 7769 (1990). 